\title{
Engagement in proactive recommendations
}

\section{The role of recommendation accuracy, information privacy concerns and personality traits}

\author{
Laurens Rook ${ }^{1} \cdot$ Adem Sabic $^{2} \cdot$ Markus Zanker $^{3}$ \\ Received: 13 November 2017 / Revised: 13 September 2018 / Accepted: 17 September 2018 / \\ Published online: 6 October 2018 \\ (C) The Author(s) 2018, corrected publication 2018
}

\begin{abstract}
The present research explored to what extent user engagement in proactive recommendation scenarios is influenced by the accuracy of recommendations, concerns with information privacy, and trait personality. We hypothesized that people's self-reported information privacy concerns would matter more when they received accurate (vs. inaccurate) proactive recommendations, because these pieces of advice would seem fair to them. We further hypothesized that this would particularly be the case for people high on the social personality trait Extraversion, who are by inclination prone to behaving in a more socially engaging manner. We put this to the test in a controlled experiment, in which users received manipulated proactive recommendations of high or low accuracy on their smartphone. Results indicated that information privacy concerns positively influenced a user's engagement with proactive recommendations. Recommendation accuracy influenced user engagement in interaction with information privacy concerns and personality traits. Implications for the design of human-computer interaction for recommender systems are addressed.
\end{abstract}

Keywords Information privacy · Concerns for information privacy · Proactive recommendation delivery · Personality-aware recommendations . Human-computer interaction

Markus Zanker

mzanker@unibz.it

Laurens Rook

1.rook@tudelft.nl

Adem Sabic

ademsabic@gmail.com

1 Delft University of Technology, Delft, Netherlands

2 Alpen-Adria-Universität Klagenfurt, Klagenfurt, Austria

3 Free University of Bozen-Bolzano, Bolzano, Italy 


\section{Introduction}

Recommender systems (RS) are automated decision support tools designed to provide custom-made advice on items to facilitate people's navigation in large product or information spaces. RS offer personalized suggestions based on presumed needs and preferences of a user and other people's behavior. They help people overcome information overload, either by providing recommendations on request, or by delivering them proactively (Jannach et al. 2010; Ricci et al. 2015). The accuracy of predictions about users' needs and preferences is a fundamental principle in RS research (Jannach et al. 2012). In RS based on the collaborative filtering $(\mathrm{CF})$ paradigm, for instance, the accuracy of recommendations derives from behavioral patterns previously disclosed by all users. A potential side-effect of accuracy as a quality metric is that the RS may recommend items too similar to what the user liked before. Such recommendations may no longer satisfy the user (Konstan and Riedl 2012; McNee et al. 2006). To deal with this issue, scholars explore other quality metrics that tap into the novelty, diversity, and unexpectedness of recommended items. Notwithstanding this, users generally appreciate recommendations based on accurate user profiles, whereas they don't like recommendations based on impersonal algorithms. Accuracy as a quality metric, therefore, remains a necessary condition; if the RS is not accurate, the user will not trust it (Jannach et al. 2016). Precisely this element of trustworthiness potentially turns accurate RS into powerful tools to deal with people's concerns with information privacy during navigation in large product or information spaces. Many online customers mistrust service providers, and fear that commercial organizations will disclose personal information without their explicit consent. Already before the advent of the smartphone, for instance, $72 \%$ of the people indicated to be afraid that external parties recorded and profiled their online behavior (Smith et al. 2011), while 85\% of the people expressed the desire to obtain more control over the ways in which firms used their personal data online (Bélanger and Crossler 2011). With the emergence of large-scale business intelligence and data analytics aimed at mobile devices, the information privacy concerns of the public at large may even further increase (Chen et al. 2012). Within the RS community, scholars increasingly take into consideration personality traits when developing user-centered RS - among others, to tailor the technology to cognitive, affective and other differences that influence user interaction with RS (Tkalcic and Chen 2015). In this work, we bring together three important factors for the design of recommendation systems: accuracy of recommendations, information privacy concerns, and personality traits. In our study, we invited participants to work on several travel planning tasks. Participants had to download these tasks via a mobile application, and to execute them on their own smartphone. Results of our experimental study show that all of the three factors positively influenced user engagement.

\section{Theory}

\subsection{Information privacy research and proactive recommendation delivery}

Information privacy refers to "the ability of the individual to personally control information about one's self" (Smith et al. 1996). Advances in information and communication technology, especially regarding the increasing pervasiveness of Web 2.0 and Web 3.0 technologies (Chen et al. 2012), have fueled a debate about the ethical aspects of information privacy (Westin 2003). People wish to be connected with external information of outstanding quality provided by external parties, yet aspire to be or remain in control of their personal 
information (Bélanger and Crossler 2011). The normative aspects of information privacy have been discussed in literature reviews: like physical privacy, information privacy is often regarded a fundamental human right (i.e., people ought to be in control over their personal information) (Smith et al. 2011; Bélanger and Crossler 2011).

There is a tension between the fundamental human right to be in control over personal information and the commercial trend towards personalization of product and service offerings. Personalization refers to "the ability to provide content and services tailored to individuals based on knowledge about their preferences and behaviors" (Adomavicius and Tuzhilin 2005). It is a crucial factor in the development and proper execution of a vendor's Customer Relationship Management (CRM) (Xu et al. 2011). In e-commerce transactions, customers often make a privacy calculus to determine, if they perceive the tradeoff between keeping or sharing their personal data with a vendor and what they would receive in return, as (un)fair. Intriguingly, customers are more willing to give away personal information when it feels convenient to them, and when they trust the vendor (Dinev and Hart 2006; Chellappa and Sin 2005). This so-called privacy-personalization paradox has been widely documented in the literature (Smith et al. 2011; Norberg et al. 2007; Dinev and Hart 2006; Chellappa and Sin 2005; Li et al. 2011; Xu et al. 2012; Ho and Tam 2006; Awad and Krishnan 2006).

A substantial part of the research into information privacy focuses on individual differences in people's concerns regarding the disclosure of personal information online (Bélanger and Crossler 2011; Li et al. 2011). This emphasis on the individual is largely due to the success of the Concern for Information Privacy (CFIP) Instrument, a 15-item scale based on four dimensions (collection, errors, secondary use, and unauthorized access), which is usually treated as an overall proxy for privacy concerns (Smith et al. 1996; Stewart and Segars 2002). The instrument measures people's concerns regarding the disclosure of personal online information without the need to actually have them give out any personal information. The CFIP thus measures ways in which people in general differ in their apprehension regarding information privacy, and such attitudes are independent from context and domain; see (Tkalcic and Chen 2015) for a similar point. The CFIP has become the gold standard in IS research for measurement of individual differences in privacy concerns (Smith et al. 2011; Bélanger and Crossler 2011).

More recently, the Internet Users' Information Privacy Concerns (IUICP) Instrument was put forward as an alternative measurement instrument. Like the CFIP, the IUIPC on an aggregate level taps into overall information privacy concerns (Malhotra et al. 2004), but from the angle of social contract theory and justice theory (Tyler 1994). It specifically assesses the perceived trade-offs between the individual, who discloses his/her own online data, and the external party, who uses the online data. An outcome is perceived as fair when the amount of online data collected by the other party is regarded as just (collection), when the information exchange can be approved or opted-out at will (control), or when it is clear that the information exchange took place, and with what effect (awareness). Similar to the CFIP, the IUIPC is a context- and domain-independent self-report measure of information privacy concerns. Where the CFIP taps into corporate-centered information exchange, the IUIPC more clearly focuses on the extent to which people value perceived fairness of information exchange (Xu et al. 2012). The IUIPC explains more variance than the CFIP (Malhotra et al. 2004), see also Bélanger and Crossler (2011), Li et al. (2011), and Xu et al. (2012).

Thus far, most research on information privacy concerns has been conducted from the perspective of technology acceptance models. In this well-known paradigm, surveys are collected to link information privacy concerns to a participant's behavioral intention to use a specific information technology (Davis 1989; Davis et al. 1989). However, results have 
been inconclusive as to the existence and direction of an association of information privacy concerns and behavioral intention to engage with technology. Many studies have predicted and found a reluctance among people high on such concerns to use information technology (Dinev and Hart 2006; Malhotra et al. 2004; Li et al. 2011; Xu et al. 2012), yet other studies have failed to replicate this negative effect (Son and Kim 2008); for a recent review, see Kokolakis (2017). For instance, a study that explored people's behavioral intention to engage on the social networking site Facebook failed to establish evidence for a negative effect of high levels of information privacy concerns on app engagement (Wisniewski et al. 2015). Under reference to King et al. (2011), the authors reasoned that most participants in their student sample probably did not know how mobile applications work; see also Kokolakis (2017).

Unfortunately, conclusions in the literature on technology acceptance models derive from self-reported behavioral intentions, not from measurement of actual user behavior (i.e., log data). Intriguingly, the limited number of studies that did collect log data, showed that users high on information privacy concerns, when downloading and engaging with a mobile application, in reality do not care about privacy issues (Buck et al. 2014; Kelley et al. 2013). Actual engagement with an IS system captures many user activities (such as searching and browsing, text writing and editing, information storage and retrieval, and engagement duration). Not all those activities appear equally troublesome from an information privacy point of view (Kokolakis 2017). Interestingly, Wisniewski et al. (2015) propose that a transparent flow of information on mobile applications may matter more for the engagement of users high on information privacy concerns. This observation is relevant to the present discussion: proactive RS may be effective, precisely because the user acknowledges the link between disclosed information and the reception of accurate recommendations. The transparency between the two will lead the user to trust and appreciate the recommendations received (Jannach et al. 2016). RS with maximum accuracy in everyday practice not always make perfect recommendations (Knijnenburg et al. 2010; Konstan and Riedl 2012; McNee et al. 2006; Jannach et al. 2016), but it nonetheless makes sense that, in some RS scenarios, a high accuracy of recommendations will lead to higher user engagement, also for people highly concerned with their privacy. Like (Wisniewski et al. 2015), this position is grounded in the literature on the role of information transparency in information exchange (Awad and Krishnan 2006).

Based on the above, we posit that the high (vs. low) information privacy concerns of a user will positively impact user engagement with a mobile application. Moreover, under reference to Awad and Krishnan (2006) and Wisniewski et al. (2015), we predict that this effect will depend on the high (vs. low) accuracy of proactively delivered recommendations. The added value of accurate RS will be so obvious to the user that also those with enhanced information privacy concerns will stop worrying, and engage in the RS. We thus hypothesize:

- Hypothesis la: The user's high (vs. low) information privacy concerns will positively influence user engagement.

- Hypothesis $1 \mathrm{~b}$ : The effect of high (vs. low) recommendation accuracy on user engagement is moderated by a user's high (vs. low) information privacy concerns.

\subsection{The five factor model of personality and proactive recommendation delivery}

Scholars in personality research explore individual differences in personality and ability. They focus on the relatively enduring ways, in which individuals differ from each other 
regarding the ways in which they think, feel, and act. Some personality psychologists develop measurement instruments to tap into theoretically grounded aspects of personality (Mischel et al. 2007). In recent years, the domain of personality psychology has come under the attention of many other academic disciplines, including those working on information and communication technologies. Among others, the measurement of individual differences in trait personality holds promise for the development of user-centered RS (Tkalcic and Chen 2015).

One of the most widely used personality theories is the five-factor model of personality, also known as the Big Five (McCrae and Costa 1987). This model assumes that a person's personality can be broken down into five hierarchical dimensions that remain stable across a lifespan. Each dimension has a psychobiological grounding, and is independent from people's age, gender, ethnicity, and social economic status (Costa and MacCrae 1992). The five factors in the model are: Openness to Experience, Conscientiousness, Neuroticism, Extraversion, and Agreeableness. Each of these overarching Big Five personality traits is composed of more specific traits. For instance, trait Openness to Experience broadly captures the extent to which someone is original, has broad interests, and is open to novel experiences and ideas. Specifically, someone high on Openness is creative, curious, imaginative and unconventional (or rather conventional, cautious). Likewise, trait Conscientiousness describes the extent to which someone is precise, careful and reliable (or rather imprecise, careless, and undependable), while trait Neuroticism captures a person's tendency to experience negative emotional states and distress (or is rather calm and even-tempered) (McCrae and John 1992).

The personality traits Agreeableness and Extraversion are different from the other three dimensions, as they represent the social dimensions of the five-factor model. Agreeableness taps into the tendency to get along with other people; it specifically captures the extent to which someone is altruistic, caring, and emotionally supportive (or rather indifferent, selfcentered, and hostile). People high on Agreeableness are often regarded as team players, as they display high levels of trust and cooperation in their social interactions (Bell 2007; Driskell et al. 2006). Extraversion, finally, is a person's tendency to experience positive emotional states and to feel good about oneself and others. It specifically taps into the extent to which someone is outgoing, cheerful, and warm (or rather quiet, timid, and withdrawn) (Costa and MacCrae 1992). Research in offline (i.e., social, work, and entrepreneurial) settings has found that people high on Extraversion more readily engage in social interactions with other people than those low on Extraversion (Bell 2007; Driskell et al. 2006; Zhao and Seibert 2006).

In RS research, scholars have become interested in the role of Big Five personality traits in user engagement. As summarized in Tkalcic and Chen (2015), the research activities roughly break down into: (1) the implicit extraction of Big Five personality traits from user data, and (2) the explicit measurement of Big Five personality traits via questionnaires. Overall, however, the evidence for, and direction of, effects of Big Five personality traits on engagement with RS is inconclusive. Regarding trait Extraversion, for instance, early studies reported negative effects of trait Extraversion on engagement, under the assumption that people high on Extraversion would favor offline social interaction over online social interaction (Amichai-Hamburger et al. 2002). The negative effect of Extraversion on user engagement is sometimes replicated (Karumur et al. 2016), but more often contradicted. Among others, people engaging in online games tend to be higher on trait Extraversion (Teng 2008). On Facebook, users high on trait Extraversion interact more with others, share more content and objects, engage in group membership more often, and significantly click more on 'likes' (Bachrach et al. 2012). Likewise, Ross et al. (2009) already identified that 
people high on Extraversion engage more heavily in group membership on Facebook, even though they did not find an impact on the number of clicks, number of Facebook friends, or time spent online. Also from a NeuroIS perspective, evidence has been generated for a causal relationship between trait Extraversion and information search behavior via an IS platform. Participants, who had filled out an extensive Big Five questionnaire first, went through a sequence of information-seeking tasks. With the help of an eye-tracker, it was found that people high on Extraversion displayed heightened engagement on a series of information search tasks (Al-Samarraie et al. 2017).

Interestingly, scholars have expressed interest in the behavioral underpinnings of information privacy concerns (Bélanger and Crossler 2011). It has, specifically, been observed that "[o]ther individual differences could be studied, such as the effects of [...] personality traits [...] on information privacy concerns. There is also a need to study the moderating effects these individual differences could have on the linkages between information privacy and dependent variables like e-business adoption" (Bélanger and Crossler 2011). Along such lines, a single correlational study tentatively explored the relation between Big Five personality traits and individual differences in privacy concerns. This study reported that individuals with enhanced self-reported Extraversion, due to their social orientation, were more concerned about the wellbeing and relationship with other people than about what happens to their personal information - that is, as long as sharing or disclosing personal information was thought to facilitate social interaction (Junglas et al. 2008). It should be noted, however, that these conclusions were merely based on associations, not on causality testing. Also, measurements on behavioral intention to use a technology, or actual user interaction with RS were not included. Still, the study points to the possibility that trait Extraversion and information privacy concerns may have an impact on user engagement in interaction.

Building on these loose threads of evidence, we maintain that Extraversion will moderate the effects of recommendation accuracy on user engagement. That is, a user high on Extraversion will display more user engagement with the RS out of a wish to make a positive contribution, whereas a user low on Extraversion will do so only when engagement seems to make sense (i.e., when the RS provides highly accurate recommendations). Moreover, under reference to Junglas et al. (2008), we posit that this two-way interaction effect will be further moderated by a user's high (vs. low) self-reported information privacy concerns. We thus hypothesize:

- Hypothesis 2a: The user's high (vs. low) Extraversion will positively influence the effect of low (vs. high) recommendation accuracy on user engagement.

- Hypothesis $2 b$ : The user's high (vs. low) information privacy concerns will moderate the interaction between high (vs. low) Extraversion and high (vs. low) recommendation accuracy on user engagement.

\section{Method}

\subsection{Participants and design}

We randomly assigned participants from the business school of a Dutch university to the experimental conditions of a Recommendation Accuracy (high, low) factorial design on User Engagement, to which self-reported scores for Information Privacy Concerns and personality traits (i.e., Extraversion) were added as covariates. The initial sample consisted of 
156 undergraduate students ( 87 men and 69 women; $M_{\text {age }}=21.17$ years, $S D=1.49$ ) enrolled in an applied statistics course. Participation was on a voluntary basis (a case assignment in the absence of monetary or course-related rewards). It was made clear from the outset that the generated data would be used for educational purposes to practice statistical techniques in aggregated, anonymized, form. The data of participants $(N=25 ; 16.03 \%)$ who reportedly did not receive proactive recommendations while working on the experiment (see below) as well as the data of participants with missing values $(N=4 ; 2.56 \%)$ were omitted. As a result, the final sample consisted of the data of 127 participants (69 men and 58 women; $M_{\text {age }}=21.22$ years, $S D=1.49$ ). The latter sample was used for the analyses reported here.

\subsection{Materials and procedure}

Participants received an email in which they were invited to participate in a voluntary case assignment dedicated to "responsible e-tourism". In this email, they were pointed to a PDF document with a detailed description made available on the course website. The email further contained the link to an online pre-survey assessing Information Privacy Concerns and personality traits (see below). Recipients were also informed how to download the smartphone application to be used in the case assignment from the Google Play or Apple App Store, depending on their mobile operating system.

The PDF document stated the research objective of the case assignment as: gaining insight into the ways in which students would use and interact with an intelligent e-tourism application on their own smartphone. To that end, the document presented the business school participants with a concise introduction into e-tourism: the practice of offering complicated, tourism-related, product-service bundles via intelligent information and communication technologies. The text discussed recommender systems in non-technical terms as intelligent software tools that proactively offer personalized suggestions how to solve a problem or need, often based on a user's known and unknown preferences (Werthner et al. 1999; Werthner and Ricci 2004). Ethical aspects and information privacy concerns were briefly addressed in relation to the potential invasiveness of recommender systems on smart devices such as the smartphone. The Five Factor Model of personality (the "Big Five") was introduced as one way in which researchers and practitioners nowadays derive preferences based on personality traits. The PDF document concluded that intelligent software tools, such as recommender systems, need to be designed with a keen eye on the ethical aspects involved in order to arrive at responsible e-tourism.

The participants were invited to download and work with a mobile e-tourism application that was developed specifically for the course. The application was entitled $e$-Tourism Challenge, and it presented participants with the following plausible - yet (at the moment of data collection) imaginary - scenario and a first challenge (labeled Task 1):

(1) Prince Harry of England has publicly announced his forthcoming wedding at Buckingham Palace, London, and set the date for .... This surely will be the event of the year, and you really wish to spend the weekend in London when this royal wedding takes place. Therefore, your task will be to find a way to travel from Delft, the Netherlands, to London, UK, on .... For Task 1 we will ask you: (1) to explore what modes of transportation (e.g., bus, train, et cetera) to select for a trip to England, and (2) how much money you are prepared to spend on this trip.

While working on Task 1 (as mapped out in the scenario above), participants had the possibility to search for information via a Search \& Browse tab in the in-application browser 
(IAB). They could write down, store, and edit findings in a Notes section before submitting their final solutions. Thus, for the first challenge (Task 1), they could, for instance, record in writing what modes of transportation they considered for their trip to London, and within what price range they were making inquiries (see Fig. 1). Also, participants had the possibility to switch between foreground and background - for instance, to search for information or a Website in another browser app, or to engage in any other activity, instead. The second challenge (Task 2) in the application built on the first one, and was introduced as follows:

(2) When in London, you need a place to stay. Therefore, your next task will be to find a place that meets the following requirements: (1) it must be located as close as possible to Buckingham Palace, and (2) you must book it from ... until .... We will ask you: (1) to report the name of the location you selected, (2) the number of stars for this location, and (3) how much money it costs you to spend the weekend at your location of choice.

Again, participants could leave and edit notes in the designated section before submitting a final solution. This time, the records in writing would probably capture the type of accommodation they considered for their stay in London, and the price range, in which they were carrying out their inquests.

Importantly, while working on Task 1, but also while working on Task 2, participants started to receive proactive recommendations in the form of a series of potentially useful hyper-links, exactly $60 \mathrm{sec}$ after their first exploration of the scenario. The accuracy of these

Fig. 1 Screenshot of the in-application dashboard

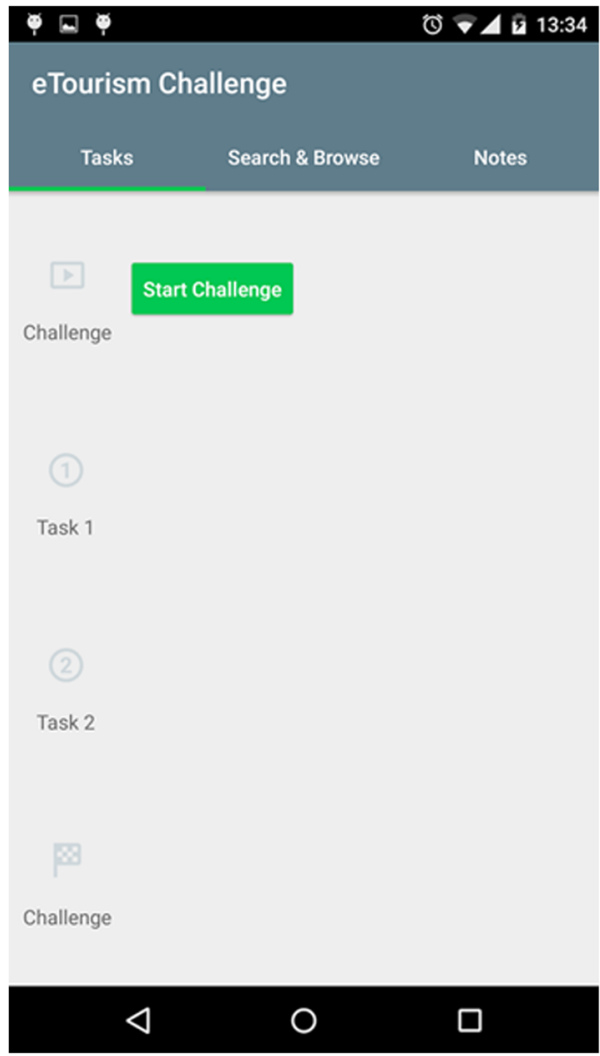


proactive recommendations was put under experimental control, meaning that it was rendered technically impossible for participants to be exposed to a mixture of and/or switches between the two experimental conditions. Participants either received a series of highly relevant, or a series of not so relevant, recommendations (see Fig. 2).

Once a participant had finished working on Tasks 1 and 2, she could submit results, after which the e-Tourism Challenge application was rendered dysfunctional. That is, the participant could no longer access the IAB, nor reinstall the application. Instead, participants were linked to an online post-survey assessing manipulation checks, their experiences with mobile application usage in general and with the current application in particular, and demographics. Participants were debriefed regarding the purpose of the study in writing and during the lectures that followed.

\subsection{Measures}

Manipulation of recommendation accuracy The accuracy of a recommender system in online experiments is typically measured by the Precision metric (signifying the share of relevant items among all items recommended) or related rank accuracy measures such as Mean Average Precision (MAP) and Normalized Discounted Cumulative Gain (nDCG) (Jannach et al. 2012; Rossetti et al. 2016). Given that study participants were anonymous first-time users of the app the relevance of recommended items was operationalized by manually selecting items that were relevant to the (static) task context of participants (Adomavicius and Tuzhilin 2015). Manipulation was induced by means of the proactive delivery of a set of recommended hyperlinks that were either very relevant or only marginally relevant for the task at hand. Specifically, participants in the high recommendation accuracy condition received a set of very relevant hyperlinks that were directly useful for solving the challenges of mode and means of travel (in Task 1) and finding an accommodation (in Task 2). People in this experimental condition thus received very handy and to-the-point links only (very precise recommendations). In contrast, participants in the low recommendation accuracy condition received a set of hyperlinks that were too generic to solve the challenges of mode and means of travel (in Task 1) and accommodation (in Task 2). People in this experimental condition thus received links that ostensibly referred to a stay in London, but they could not really contribute to solving the challenges posed since they were not relevant (i.e. low Precision) for the participants' task context (see Fig. 2).

Information privacy concerns We used the original Internet Users' Information Privacy Concerns (IUIPC) scale (Malhotra et al. 2004) to measure self-reported differences in our participant's Information Privacy Concerns. The IUIPC construct derives from a tenitem scale, which is based on three first-order dimensions labeled Control, Collection, and Awareness. Even though this leaves room for an analysis of each of these first-order dimensions in isolation, the scale was developed to assess IUIPC as an overarching second-order construct. Table 1 provides each of the ten items in the IUIPC, and shows the results of a Principal Component Analysis. Consistent with the literature (Malhotra et al. 2004), a free Promax Rotation acknowledged the multidimensionality of the scale, while clearly confirming that all items load on a single, overarching, dimension. Each item was assessed on a 7-point scale anchored at 1 (strongly disagree) and 7 (strongly agree). The second-order scale for overall IUIPC was reliable; Cronbach's $\alpha=.89$ ).

Extraversion Extraversion is one of the social dimensions in the Five Factor Model of personality (or Big Five). Many assessment instruments have been developed to measure 


\section{ㅁ. है}

We analyzed your information needs, and here are some websites that might help you.

\section{Rome2rio (Multiple)}

http://www.rome2rio.com/s/Delft/London

thetrainline.com (Trains)

http://www.thetrainline-europe.com/plan/london-to-...

$$
\text { GoEuro (Trains) }
$$

http://www.goeuro.com/trains_from_delft_to_london

$$
\text { Google Maps (Multiple) }
$$

https://www.google.co.uk/maps/dir/Delft,+Netherlan.

$$
\text { CapitaineTrain (Trains) }
$$

http://timetable.capitainetrain.com/trains/delft/l.
We analyzed your information needs, and here are some websites that might help you.

Fodors - London Travel Guide http://www.fodors.com/world/europe/england/london

TripAdvisor - Best of London Travel Guide http://www.tripadvisor.com/Tourism-g186338-London_...

LonelyPlanet - Introducing London

http://www.lonelyplanet.com/england/london

Wikitravel - London Travel Guide http://wikitravel.org/en/London

VisitBritain - London Travel Guide http://www.visitbritain.com/en/Destinations-and-Ma..
Save links to Notes

\section{Close}

Close \& Don't show anymore

\section{$\triangleleft$}

(요웅

We analyzed your information needs, and here are some websites that might help you.

Trivago - Hotels in London http://www.trivago.co.uk/

Booking.com Hotels

http://www.booking.com/city/gb/london.html

TripAdvisor Hotels

http://www.tripadvisor.com/Hotels

Google Hotel Finder

https://www.google.co.uk/hotels/

$$
\text { AirBnB }
$$

https://www.airbnb.co.uk/s/London-United-Kingdom

\section{Save links to Notes}

Close

Close \& Don't show anymore

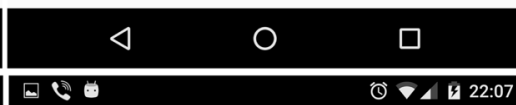

We analyzed your information needs, and here are some websites that might help you.

OpenTable - Restaurants in London http://www.opentable.co.uk/london-restaurants

TripAdvisor - Restaurants in London http://www.tripadvisor.co.uk/Restaurants-g186338-L...

Transport for London - Timetable

https://tfl.gov.uk/travel-information/timetables/

$$
\text { Search for Taxis in London }
$$

https://www.google.com/\#q=taxi+london\&rfff=1\&tbm=...

$$
\text { Yelp - Cafes in London }
$$

http://www.yelp.co.uk/search?find_desc=Cafes\&find_...

\section{Save links to Notes}

\section{Close}

Close \& Don't show anymore

\section{Save links to Notes}

\section{Close}

Close \& Don't show anymore

Fig. 2 Screenshots of recommendations, top left: high accuracy for Task 1, top right: low accuracy for Task 1, bottom left: high accuracy for Task 2, bottom right: low accuracy for Task 2 
Table 1 Factor loadings for principal component analysis with promax rotation $(N=127)$

3-Factor Solution

Item

I

II

III

Information Privacy Concerns

Consumer online privacy is really a matter of consumers'

right to exercise control and autonomy over decisions

about how their information is collected, used, and shared

Consumer control of personal information lies at the heart

of consumer privacy

I believe that online privacy is invaded when control is lost

or unwillingly reduced as a result of a marketing transaction

It usually bothers me when online companies ask me for

personal information

When online companies ask me for personal information I

sometimes think twice before providing it

It bothers me to give personal information to so many

online companies

I'm concerned that online companies are collecting

too much personal information about me

Companies seeking information online should disclose the

way the data are collected, processed, and used

A good consumer online privacy policy should have a

clear and conspicuous disclosure

It is very important to me that I am aware and knowledgeable

about how my personal information will be used

Extraversion

Am the life of the party

Don't talk a lot (reverse-scored)

Talk to a lot of different people at parties

Keep in the background (reverse-scored)

Note. Factor loadings $>.40$ are in italics

individual differences in Big Five personality traits. In the present research, we opted for the Mini-IPIP Scales (Donnellan et al. 2006) - a widely used, highly condensed and psychometrically valid version of the larger International Personality Item Pool - Five Factor Model (Goldberg 1999). Table 1 provides each of the four items in the Mini-IPIP Scales that assess trait Extraversion. Each item was measured on a 5-point scale anchored at 1 (very inaccurate) and 5 (very accurate). The overall score was computed after reverse coding of the respective items. Again, the results of a Principal Component Analysis confirmed the internal consistency of the Extraversion scale. The scale was reliable; Cronbach's $\alpha=.77$ ).

User engagement It is well-documented in the literature on organizational behavior that people characterized by higher levels of task engagement typically refrain from submitting bottom-line results, but spend time and energy in handing in decent solutions to a challenge 
(Rich et al. 2010), also in computer-mediated settings (Ray et al. 2014). In accordance to this literature, we operationalized user engagement in the present study in two different ways:

Engagement clicks In the IAB, participants could (re-)read the scenarios and descriptions for Tasks 1 and 2, search and browse for information within the application environment or via another search engine, and write down, store, and edit their findings. Engagement Clicks was therefore operationalized as the sum of all editing, description reading, and searching activities, including all switches between foreground and background.

Engagement duration Engagement Duration was operationalized as the total duration of a participant's interaction with the IAB. Engagement Duration derived from the time it took a participant (in sec) to complete Tasks 1 and 2 - thus, from the first exploration of the scenarios in the IAB until the moment in time when the solutions were submitted, and the e-Tourism Challenge was rendered dysfunctional.

Manipulation check So as to capture the extent to which participants had perceived the accuracy of the proactively delivered recommendations in congruence with experimental conditions, we used the following four items: "The travel solutions I produced for the e-Tourism Challenge were of good quality", "Overall, I found it helpful to receive the recommended set of links for the e-Tourism Challenge", "I was generally pleased with the recommended set of links I got for the e-Tourism Challenge" and "The recommended set of links for the e-Tourism Challenge enabled me to submit high-quality travel solutions", on a 7-point scale anchored at 1 (not at all true for me) and 7 (very true for me; Cronbach's $\alpha=.83)$.

\section{Results}

\subsection{Manipulation check}

We conducted a linear regression analysis on the four items of the manipulation check for recommendation accuracy. The analysis yielded a significant main effect of low versus high recommendation accuracy, $\beta=.60, t(126)=2.67, p<.01$, indicating that participants indeed had experienced the quality of the proactive recommendations they had received in congruence with their experimental condition. The manipulation therefore had been effective.

\subsection{Sample characteristics, correlations and overall distributions}

Table 2 shows that the majority of smartphone users in the present study worked with iOS rather than Android as their operating system. The other descriptives in the table derive from a list of covariate items that are more often collected in studies on information privacy concerns (Malhotra et al. 2004; Smith et al. 1996). We modified these items to tailor them to mobile app usage - so as to explore the familiarity of our student sample with mobile applications in real life. Our participants turned out to be seasoned app users: the vast majority of users in our sample had many years of experience in the usage of mobile applications on their smartphones, and were familiar with the phenomena of privacy invasions and information misuse that are often associated with information privacy concerns. They heard or read 
Table 2 Sample descriptions

\begin{tabular}{|c|c|c|}
\hline Sample descriptions & Summary & $N($ Total $=127)$ \\
\hline \multirow{8}{*}{$\begin{array}{l}\text { Experience with mobile } \\
\text { app usage }\end{array}$} & Less than 1 year & 5 \\
\hline & Less than 2 years & 1 \\
\hline & Less than 3 years & 5 \\
\hline & Less than 4 years & 18 \\
\hline & Less than 5 years & 34 \\
\hline & Less than 6 years & 29 \\
\hline & Less than 7 years & 14 \\
\hline & More than 7 years & 21 \\
\hline \multirow{5}{*}{$\begin{array}{l}\text { Falsified information } \\
\text { for mobile app usage }\end{array}$} & Never & 16 \\
\hline & Under $25 \%$ of the time & 56 \\
\hline & Between $26 \%-50 \%$ of the time & 34 \\
\hline & Between $51 \%-75 \%$ of the time & 13 \\
\hline & Over $75 \%$ of the time & 8 \\
\hline \multirow{7}{*}{$\begin{array}{l}\text { Experience with privacy invasions } \\
\text { during mobile app usage }\end{array}$} & Very infrequently & 35 \\
\hline & Infrequently & 43 \\
\hline & Somewhat infrequently & 15 \\
\hline & Neither infrequently nor frequently & 15 \\
\hline & Somewhat frequently & 16 \\
\hline & Frequently & 1 \\
\hline & Very frequently & 2 \\
\hline \multirow{7}{*}{$\begin{array}{l}\text { Heard or read about information } \\
\text { misuse with mobile apps }\end{array}$} & Not at all true of me & 7 \\
\hline & Not true of me & 10 \\
\hline & Somewhat true of me & 21 \\
\hline & Neither true or not true of me & 17 \\
\hline & Somewhat true of me & 42 \\
\hline & True of me & 24 \\
\hline & Very true of me & 6 \\
\hline \multirow[t]{2}{*}{ Operating system } & Android & 35 \\
\hline & iOS & 92 \\
\hline
\end{tabular}

$N=127$

about it, but overall encountered such privacy invasions (very) infrequently in their own life (Awad and Krishnan 2006).

Table 3 shows the descriptive statistics and a traditional correlation matrix for the factors in the experimental design, including the arithmetic means and standard deviations for Engagement Clicks and Engagement Duration. First, Extraversion appeared not to significantly correlate with the other variables in the study. Second, Recommendation Accuracy was significantly and positively correlated with overall IUIPC. Also, overall IUIPC was significantly and positively correlated with Engagement Clicks and Engagement Duration. Finally, Engagement Clicks and Engagement Duration were significantly and positively correlated - in the absence of any multicollinearity. 
Table 3 Descriptive statistics and correlations

\begin{tabular}{|c|c|c|c|c|c|c|c|c|}
\hline & & $M$ & $S D$ & 1. & 2. & 3. & 4. & 5 . \\
\hline 1. & Recommendation Accuracy & .51 & .50 & - & & & & \\
\hline 2. & IUIPC & 5.05 & 0.95 & $.19^{*}$ & - & & & \\
\hline 3. & Extraversion & 3.41 & 0.78 & -.04 & .08 & - & & \\
\hline 4. & Engagement Clicks & 16.43 & 8.91 & .01 & $.27^{* *}$ & .07 & - & \\
\hline 5. & Engagement Duration & 41269.41 & 129423.05 & .08 & $.33^{* * *}$ & .07 & $.30^{* *}$ & - \\
\hline
\end{tabular}

Note: $N=127 ; * p<.05$ level, $* * p<.01$ level, $* * * p<.001$ level; two-tailed

However, it should be emphasized that the two dependent variables in the present study, Engagement Clicks and Engagement Duration, were count variables. Count variables, among others, refer to a number of occurrences or an enumeration of events, or to series of events that occur over a period of time. They often contain zeros and low values (i.e., low arithmetic means), and tend to be skewed in terms of distribution (Cameron and Trivedi 2013; Hilbe 2014; Coxe et al. 2009). It has been argued that arithmetic means, then, may lead to biased interpretation, something that can be compensated for by plotting the observed distribution of the count variables in question (Coxe et al. 2009; Aiken et al. 2015). Figures $3 \mathrm{a}$ and $4 \mathrm{a}$, therefore, show the overall count distributions of Engagement Clicks and Engagement Duration, clearly confirming that our dependent variables must be treated as count variables. Also provided are boxplot count distributions of Engagement Clicks and Engagement Duration, split by low and high levels of Recommendation Accuracy (see Figs. $3 b$ and $4 b)$.

\subsection{Engagement clicks}

We used the General Linear Models (GLMs) module in the SPSS statistics software package to evaluate our hypotheses for Engagement Clicks, the first dependent variable in our study. As outlined in Coxe et al. (2009) and Aiken et al. (2015), the general linear model (GLM) is a larger set of regression models specifically developed for the analysis of count data. The GLM module allows for analyzing various types of count data with standard Poisson regression and alternatives, depending on the nature and extent of skewness of the distribution. As visible in Fig. 3a, a negative binomial regression was the most appropriate analysis,

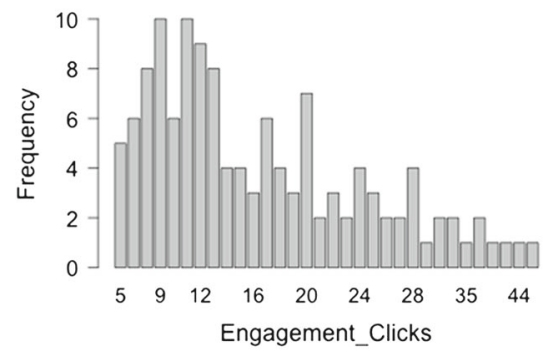

(a) Overall distribution, $n=127$ cases, both Recommendation Accuracy conditions pooled.

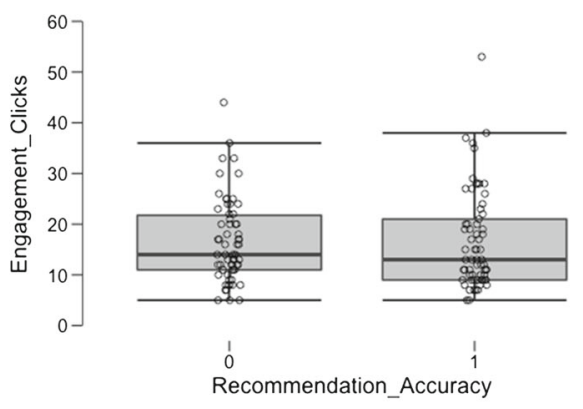

(b) Distribution per conditions, low Recommendation Accuracy condition $=0$, high Recommendation Accuracy condition $=+1$.

Fig. 3 Histogram and box plot for the engagement measure based on users' clicks 


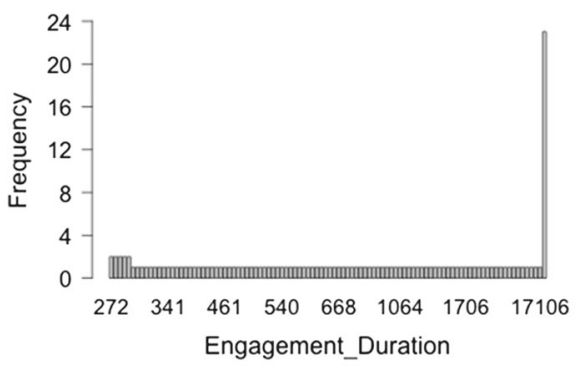

(a) Overall distribution, $n=127$ cases, both Recommendation Accuracy conditions pooled.

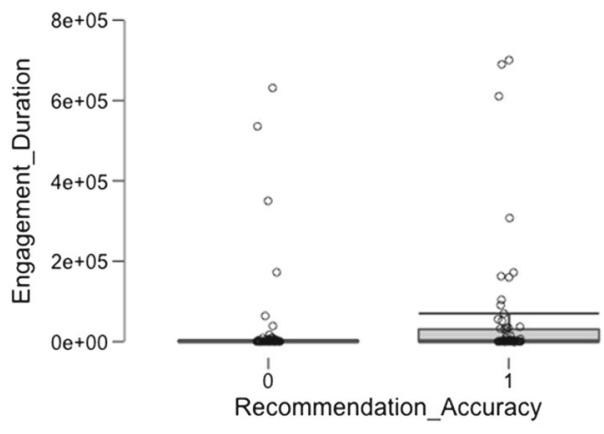

(b) Distribution per conditions, low Recommendation Accuracy condition $=0$, high Recommendation Accuracy condition $=+1$.

Fig. 4 Histogram and box plot for the engagement measure based on duration

due to a variance of the dependent variable Engagement Clicks larger than the mean (i.e., overdispersion; Coxe et al. 2009). For our analyses, we ran a negative binomial regression, with an adjusted overdispersion parameter $\phi=.17, S E=.05,95 \%$ CI $[0.35,0.55]$.

Following the procedure outlined in Aiken et al. (1991), Coxe et al. (2009), and Aiken et al. (2015), we dummy-coded our experimental factor, Recommendation Accuracy, into -0.5 and +0.5 (for low vs. high recommendation accuracy, respectively), and centered the measures of IUIPC and Extraversion at the grand mean. Next, we computed the crossproducts for the higher-order interactions between those predictors on Engagement Clicks, and entered all of these interaction terms into the equation together with the main effects to test for causality. Thus, we put to the test a full-factorial negative binomial regression model with seven effects.

We tested our full-factorial model against an intercept-only model. The Likelihood Ratio chi-square for the full negative binomial model was $\chi^{2}(7)=15.61, p<.03$, with a model deviance $D=127.09$, which showed that our fitted model led to a significant reduction in deviance, with goodness of fit values for the Log Likelihood function $=-432.93$, Akaike's Information Criterion $(\mathrm{AIC})=883.85$, the Bayesian Information Criterion $(\mathrm{BIC})=909.45$.

Table 4 summarizes the results of the negative binomial regression analysis. Confirming our Hypothesis 1a, overall IUIPC had a significant positive effect on the log odds of

Table 4 Final model estimates of fixed and interaction effects on engagement clicks

\begin{tabular}{|c|c|c|c|c|c|}
\hline \multirow[b]{2}{*}{ Model effects } & \multirow[b]{2}{*}{ Est. } & \multicolumn{2}{|l|}{ Std. } & \multicolumn{2}{|l|}{ Wald } \\
\hline & & error & $d f$ & $\chi^{2}$ & $p$ \\
\hline Intercept & 2.75 & 0.06 & 1 & 2014.98 & $<.0001$ \\
\hline Recommendation Accuracy & 0.06 & 0.09 & 1 & 0.54 & $n s$. \\
\hline IUIPC & 0.23 & 0.07 & 1 & 11.71 & $<.001$ \\
\hline Extraversion & -0.03 & 0.08 & 1 & 0.16 & $n s$. \\
\hline Recommendation Accuracy x IUIPC & -0.13 & 0.09 & 1 & 1.83 & $n s$. \\
\hline Recommendation Accuracy x Extraversion & 0.11 & 0.11 & 1 & 0.93 & $n s$. \\
\hline IUIPC x Extraversion & 0.08 & 0.09 & 1 & 0.75 & $n s$. \\
\hline Recommendation Accuracy x IUIPC x Extraversion & -0.02 & 0.13 & 1 & 0.02 & $n s$. \\
\hline
\end{tabular}


Engagement Clicks, Wald $\chi^{2}(1)=11.71, p<.001$, indicating that people high on information privacy concerns produced a higher number of clicks in the IAB than those low on information privacy concerns. That is, people high on overall IUIPC engaged more heavily in editing, reading, and searching activities, as well as in switching between foreground and background. No other significant effects were revealed.

\subsection{Engagement duration}

For Engagement Duration, the second dependent variable in our study, we again relied on the GLM module in SPSS. As shown in Fig. 4a, the count distribution for Engagement Duration was characterized by a partly exponential shape, which deviated from the partly linear form of standard generalized linear models. It is more appropriate to analyze such exponential dispersion models or Tweedie distributions (Jorgensen 1987) with the help of mixed count regressions. We ran a Tweedie mixed count regression with a maximum likelihood estimate $\theta=207.63, S E=14.29,95 \%$ CI [181.43, 237.61] to evaluate our hypotheses for Engagement Duration.

We applied the same procedure for building regression models with fixed and interaction effects as before (Aiken et al. 1991; Coxe et al. 2009; Aiken et al. 2015), meaning that we tested the same full-factorial regression model with seven effects. Our full-factorial model was tested against an intercept-only model. The Likelihood Ratio chi-square for the full model was $\chi^{2}(7)=137.96, p<.0001$, with a model deviance $D=337.23$, which showed that our fitted model led to a significant reduction in deviance, with goodness of fit values for the Log Likelihood function $=-1481.04$, Akaike's Information Criterion (AIC) $=$ 2980.07, the Bayesian Information Criterion (BIC) $=3005.67$.

Table 5 summarizes the regression results. Again, in confirmation of Hypothesis 1a, overall IUIPC had a significant positive effect on the log odds of Engagement Duration, Wald $\chi^{2}$ $(1)=11.71, p<.001$; people high on IUIPC interacted longer with the IAB than those low on IUIPC. In support of Hypothesis $1 \mathrm{~b}$, the log odds of Engagement Duration as a function of IUIPC were dependent on Recommendation Accuracy, Wald $\chi^{2}(1)=6.58, p<.01$. People high (vs. low) on IUIPC interacted longer with the IAB in general, but especially when Recommendation Accuracy was high. This set them apart from the lack of engagement of people low on IUIPC. Confirming our Hypothesis 2a, the log odds of Engagement Duration as a function of Extraversion were dependent on Recommendation Accuracy, Wald $\chi^{2}(1)=$ $5.02, p<.03$. People high on Extraversion generally displayed high Engagement Duration,

Table 5 Final model estimates of fixed and interaction effects on engagement duration

\begin{tabular}{|c|c|c|c|c|c|}
\hline \multirow[b]{2}{*}{ Model effects } & \multirow[b]{2}{*}{ Est. } & \multicolumn{2}{|l|}{ Std. } & \multicolumn{2}{|l|}{ Wald } \\
\hline & & error & $d f$ & $\chi^{2}$ & $p$ \\
\hline Intercept & 9.75 & 0.18 & 1 & 3002.50 & $<.0001$ \\
\hline Recommendation Accuracy & 0.21 & 0.24 & 1 & 0.74 & $n s$. \\
\hline IUIPC & 1.54 & 0.16 & 1 & 92.89 & $<.0001$ \\
\hline Extraversion & -0.10 & 0.26 & 1 & 0.16 & $n s$. \\
\hline Recommendation Accuracy x IUIPC & -0.61 & 0.24 & 1 & 6.58 & $<.01$ \\
\hline Recommendation Accuracy x Extraversion & 0.77 & 0.35 & 1 & 5.02 & $<.03$ \\
\hline IUIPC $x$ Extraversion & 0.15 & 0.28 & 1 & 0.28 & $n s$. \\
\hline Recommendation Accuracy x IUIPC x Extraversion & -0.02 & 0.36 & 1 & 0.02 & $n s$ \\
\hline
\end{tabular}


whereas those low on Extraversion did so only when Recommendation Accuracy was high. We did not find any evidence that this interaction effect of Recommendation Accuracy and Extraversion on Engagement Duration was further moderated by IUIPC. Hypothesis $2 b$, therefore, had to be rejected. No other significant effects were revealed.

\section{Discussion}

The present study explored to what extent the engagement of users in proactive RS was influenced by the accuracy of recommendations, their concerns with information privacy, and differences in trait personality. The contributions of our research are as follows:

Scientific relevance First, our work offers refinement to the already vast literature on the role of information privacy concerns in user engagement with IS (Smith et al. 2011; Bélanger and Crossler 2011; Dinev and Hart 2006). Our results especially contribute to the growing awareness that information privacy concerns may exert a positive impact on user engagement (Buck et al. 2014; Kelley et al. 2013; Kokolakis 2017). That such a positive effect indeed exists for clicks and duration, two different measures of user engagement, is the main insight from our study. This empirically confirms a possibility raised in Wisniewski et al. (2015), and adds to the growing popularity of the Internet Users' Information Privacy Concerns (IUIPC) measure (Malhotra et al. 2004) for information privacy concerns (Li et al. 2011; Xu et al. 2012). Our work further hints towards the possibility that accurate RS can compensate for a lack of user engagement due to high information privacy concerns. Even though recommendation accuracy did not lead to higher user engagement for all participants, our results revealed that people high on information privacy concerns engage even more with the RS. This result squares with a growing literature on the benefits of information transparency on engagement with IS (Awad and Krishnan 2006; Jannach et al. 2016). A similar trend due to recommendation accuracy was observed for users low on trait Extraversion, who also engaged longer with the RS when recommended items were highly accurate. Theoretically, this is in line with prior separate findings on trait personality in IS settings (Junglas et al. 2008; Al-Samarraie et al. 2017). However, it remains to be seen to what extent each of the observed trends counter or rather confirm criticism from within the RS community, that users typically display more engagement and satisfaction with RS, not because the recommendations they receive are objectively of higher quality, but because they are merely subjectively perceived as such (Knijnenburg et al. 2010).

Limitations We should, in that respect, hasten to acknowledge the limitations of this research. First of all, the results derive from a single study. Of course, generalization should not be based on the results of single studies (Driskell and Salas 1992). Second, the small size of our experiment did not allow for the provision of more robust evidence for our findings. We specifically lacked the statistical power to provide a thorough analysis of the trends in personality traits that were observed and reported in this research (see also our discussion of future work below). Moreover, the participants in our sample were taken from the classroom. Even though college student samples are typically used in research on the Big Five, cf. John and Srivastava (1999), it has been widely acknowledged in general (Cozby and Bates 2012) and for Big Five personality traits in particular (Rammstedt et al. 2010) that findings from college student samples do not fully generalize to the wider population. Also related to the issue of external validity, a large percentage of participants in our sample reported to have infrequently or very infrequently experienced privacy invasions during 
mobile application usage (see Table 2). On the one hand, this confirms a trend observed elsewhere that younger people are less concerned with privacy issues than older generations (Hassing 2015). However, it also illustrates that our findings may not entirely generalize to other user groups such as older demographics. Further, we kept the factor recommendation accuracy under experimental control, but the same cannot be said for our participants. They had full freedom to download, explore, interact, or ignore the software at their convenience - i.e., as long as no task results had been submitted. This may have led some of our participants to procrastinate more than typically allowed for in the heavily controlled setting of the laboratory. Naturally, this procedure does not meet the requirements of a full-fledged laboratory experiment, but added to the ecological validity of our study (Mook 1983). Finally, user engagement in the setting of proactive RS seldom is a one-shot event, but rather a series of interactions at various intervals. The present setup did not fully accommodate users in that respect. Users could not really learn from experience, and come to trust the RS on its merits, as the mobile application was rendered dysfunctional, once the participant had submitted results to all challenges. This is something that should be taken into consideration in future iterations of our RS.

Future work We did not explore all Big Five personality dimensions in our study. This constitutes both a weakness and an opportunity for future research, as the personality dimensions Openness, Conscientiousness, Neuroticism, and Agreeableness may also impact user engagement. The RS literature reveals mixed, and often contradictory, evidence for the role of these other Big Five dimensions on user engagement. For instance, the positive effects of Openness and Conscientiousness on online game engagement (Teng 2008) are only partially confirmed for user engagement in Facebook (for Openness, but Conscientiousness produces mixed effects) (Bachrach et al. 2012), while they yield (negative) effects for Conscientiousness and no effects for Openness in a study on engagement in diverse RS (Chen et al. 2013). It would be interesting to explore in future research how Openness and Conscientiousness, in tandem with information privacy concerns, impact user engagement in proactive recommendations. Probably, users high on Openness, due to their dispositional curiosity and eagerness to explore novel stimuli, would engage with a system regardless of the accuracy of the recommendations or information privacy concerns. Users high on Conscientiousness (i.e, precision), on the other hand, would likely engage heavily in a system offering accurate recommendations (Al-Samarraie et al. 2017). This level of user engagement could be further enhanced by someone's information privacy concerns; see Junglas et al. (2008). This association with information privacy concerns may not exist for people high on Neuroticism, however (Junglas et al. 2008). Such users, characterized by heightened negative emotional states and distress, would preferably not engage in a system offering recommendations that are inaccurate - hence, causing distress. On the other hand, and more counterintuitive, low-quality recommendations may also cause the neurotic user to engage more with the system. People high on Neuroticism often are anxious not to make mistakes and, as a consequence, invest substantial cognitive effort in exploring and correcting errors (Perkins 2014). Proactive suggestions of low quality may cause the neurotic user to spend more time in error detection and error correction. Future work could attempt to shed light on these and other issues related to the Big Five.

Moreover, Extraversion is not the only social dimension in the Big Five framework: Agreeableness is the second. We did collect trait Agreeableness scores for our sample, but, due to our small sample size, we could not include those scores in our regression models (we lacked statistical power to meaningfully produce a full factorial model with fifteen effects). Nevertheless, tentative exploration revealed that Agreeableness was positively and 
moderately correlated with Extraversion $(r=.30, p<.0001)$. Full factorial GLM analyses of those fifteen effects on our two user engagement factors seemed to hint towards a pattern, in which the two social dimensions positively reinforce one another, and, in interaction, produce an uplifting effect on user engagement. These results probably stem from an overfitted model, thus we should be cautious to draw conclusions. Still, this issue could be addressed in greater detail in future study. Prior work in non-IS domains has reported robust personality configurations with high Extraversion and Agreeableness clusters, as well as with low Extraversion and Agreeableness clusters (Rentfrow et al. 2013). Collection of much larger, and preferably independent, samples of people working with the e-Tourism Challenge would, therefore, offer the opportunity to model personality trait clusters together with information privacy concerns on a larger scale.

Yet, another avenue of future work would be to adopt in follow-up research endeavors some of the more recent insights from social justice research (Tyler 1994). So far, scholars on information privacy concerns primarily relied on self-report measures like the IUIPC to capture (un)fairness. Remarkably, the research domain of social justice has also proven successful in the development of experimental paradigms for business ethics that temporarily induce people with (un)fairness levels in the safety of the laboratory (De Cremer et al. 2011), as well as in a more realistic, ecologically valid, IS context (De Cremer et al. 2017). In that light, some promising work in the absence of RS has mapped the neural correlates of decision-making under fair vs. unfair circumstances. Especially aversively motivated people seem to respond strongly to situations in which they feel obliged to accept an unfair offer (Servaas et al. 2015). It thus makes intuitive sense to postulate that information privacy concerns in RS have a neural basis rooted in aversive motivation. Future study could seek to explicitly put this to the test, and to explore to what extent user engagement in proactive $\mathrm{RS}$ will be triggered by the anticipated (un)fairness of RS in some users.

\section{Conclusions}

In conclusion, the present research highlights the need to account for a user's information privacy concerns and personality traits in the development of proactive, accurate, RS. More generally, this work points to the importance of controlling for anticipated fairness in the design of recommender systems.

Funding Information This work was supported by the Open Access Publishing Fund provided by the Free University of Bozen-Bolzano.

Open Access This article is distributed under the terms of the Creative Commons Attribution 4.0 International License (http://creativecommons.org/licenses/by/4.0/), which permits unrestricted use, distribution, and reproduction in any medium, provided you give appropriate credit to the original author(s) and the source, provide a link to the Creative Commons license, and indicate if changes were made.

\section{References}

Adomavicius, G., \& Tuzhilin, A. (2005). Personalization technologies: a process-oriented perspective. Communications of the ACM, 48(10), 83-90.

Adomavicius, G., \& Tuzhilin, A. (2015). Context-aware recommender systems, (pp. 191-226). Boston: Springer.

Aiken, L.S., Mistler, S.A., Coxe, S., West, S.G. (2015). Analyzing count variables in individuals and groups: Single level and multilevel models. Group Processes \& Intergroup Relations, 18(3), 290-314. 
Aiken, L.S., West, S.G., Reno, R.R. (1991). Multiple regression: Testing and interpreting interactions Sage.

Al-Samarraie, H., Eldenfria, A., Dawoud, H. (2017). The impact of personality traits on users' informationseeking behavior. Information Processing \& Management, 53(1), 237-247.

Amichai-Hamburger, Y., Wainapel, G., Fox, S. (2002). On the internet no one knows i'm an introvert: extroversion, neuroticism, and internet interaction. Cyberpsychology \& Behavior, 5(2), 125-128.

Awad, N., \& Krishnan, M. (2006). The personalization privacy paradox: an empirical evaluation of information transparency and the willingness to be profiled online for personalization. MIS Quarterly, 30, $13-28$.

Bachrach, Y., Kosinski, M., Graepel, T., Kohli, P., Stillwell, D. (2012). Personality and patterns of facebook usage. In Proceedings of the 4th Annual ACM Web Science Conference, pp. 24-32. ACM.

Bell, S.T. (2007). Deep-level composition variables as predictors of team performance: a meta-analysis. Journal of Applied Psychology, 92(3), 595.

Bélanger, F., \& Crossler, R. (2011). Privacy in the digital age: a review of information privacy research in information systems. MIS Quarterly, 35(4), 1017-1041.

Buck, C., Horbel, C., Germelmann, C.C., Eymann, T. (2014). The unconscious app consumer: discovering and comparing the information-seeking patterns among mobile application consumers. In Proceedings of the 2014 European Conference on Information Systems (ECIS2014).

Cameron, A.C., \& Trivedi, P.K. (2013). Regression analysis of count data, vol. 53. Cambridge University Press.

Chellappa, R.K., \& Sin, R.G. (2005). Personalization versus privacy: an empirical examination of the online consumer's dilemma. Information Technology and Management, 6(2), 181-202.

Chen, H., Chiang, R., Storey, V. (2012). Business intelligence and analytics: From big data to big impact. MIS Quarterly, 36, 1165-1188.

Chen, L., Wu, W., He, L. (2013). How personality influences users' needs for recommendation diversity? CHI'13 Extended abstracts on human factors in computing systems, pp. 829-834. ACM.

Costa, P.T., \& MacCrae, R.R. (1992). Revised NEO personality inventory (NEO PI-r) and NEO five-factor inventory (NEO-FFI): professional manual. Psychological Assessment Resources, Incorporated.

Coxe, S., West, S.G., Aiken, L.S. (2009). The analysis of count data: a gentle introduction to poisson regression and its alternatives. Journal of Personality Assessment, 91(2), 121-136.

Cozby, P.C., \& Bates, S.C. (2012). Methods in behavioral research. New Age Int. Publishers.

Davis, F.D. (1989). Perceived usefulness, perceived ease of use, and user acceptance of information technology. MIS Quarterly, 13(3), 319-340.

Davis, F.D., Bagozzi, R.P., Warshaw, P.R. (1989). User acceptance of computer technology: a comparison of two theoretical models. Management Science, 35(8), 982-1003.

De Cremer, D., van Dick, R., Tenbrunsel, A., Pillutla, M., Murnighan, J.K. (2011). Understanding ethical behavior and decision making in management: a behavioural business ethics approach. British Journal of Management, 22, S1-S4.

De Cremer, D., Nguyen, B., Simkin, L. (2017). The integrity challenge of the internet-of-things (iot): on understanding its dark side. Journal of Marketing Management, 33(1-2), 145-158.

Dinev, T., \& Hart, P. (2006). An extended privacy calculus model for e-commerce transactions. Information Systems Research, 17(1), 61-80.

Donnellan, M.B., Oswald, F.L., Baird, B.M., Lucas, R.E. (2006). The mini-ipip scales: Tiny-yet-effective measures of the big five factors of personality. Psychological Assessment, 18(2), 192.

Driskell, J.E., Goodwin, G.F., Salas, E., O'Shea, P.G. (2006). What makes a good team player? personality and team effectiveness. Group Dynamics: Theory Research, and Practice, 10(4), 249.

Driskell, J.E., \& Salas, E. (1992). Can you study real teams in contrived settings? the value of small group research to understanding teams. In: R. W. Swezey \& E. Salas (Eds.), Teams: Their training and performance pp. 101-126.

Goldberg, L.R. (1999). A broad-bandwidth, public domain, personality inventory measuring the lower-level facets of several five-factor models. Personality Psychology in Europe, 7(1), 7-28.

Hassing, L. (2015). An exploratory study in the concerns for information privacy: finding a trend factors of influence and spheres of informational justice. Thesis at the Faculty of Technology, Policy and Management, TU Delft.

Hilbe, J.M. (2014). Modeling count data. Cambridge University Press.

Ho, S.Y., \& Tam, K. (2006). Understanding the impact of web personalization on user information processing and decision outcomes. MIS Quarterly, 30(4), 865-890.

Jannach, D., Resnick, P., Tuzhilin, A., Zanker, M. (2016). Recommender systems: Beyond matrix completion. Communications of the ACM, 59(11), 94-102.

Jannach, D., Zanker, M., Felfernig, A., Friedrich, G. (2010). Recommender systems: an introduction. Cambridge University Press. 
Jannach, D., Zanker, M., Ge, M., Gröning, M. (2012). Recommender systems in computer science and information systems - a landscape of research. In Proceedings 13th International Conference on E-commerce and web technologies (EC-Web) (pp. 76-87).

John, O.P., \& Srivastava, S. (1999). The big five trait taxonomy: history, measurement, and theoretical perspectives. Handbook of personality: Theory and research, 2, 102-138.

Jorgensen, B. (1987). Exponential dispersion models. Journal of the Royal Statistical Society. Series B (Methodological), pp. 127-162.

Junglas, I.A., Johnson, N.A., Spitzmüller, C. (2008). Personality traits and concern for privacy: an empirical study in the context of location-based services. European Journal of Information Systems, 17(4), 387402.

Karumur, R.P., Nguyen, T.T., Konstan, J.A. (2016). Exploring the value of personality in predicting rating behaviors: a study of category preferences on movielens. In Proceedings of the 10th ACM Conference on Recommender Systems, pp. 139-142. ACM.

Kelley, P.G., Cranor, L.F., Sadeh, N. (2013). Privacy as part of the app decision-making process. In Proceedings of the SIGCHI Conference on Human Factors in Computing Systems, pp. 3393-3402. ACM.

King, J., Lampinen, A., Smolen, A. (2011). Privacy: is there an app for that? In Proceedings of the 7th Symposium on Usable Privacy and Security, p. 12. ACM.

Knijnenburg, B.P., Willemsen, M.C., Hirtbach, S. (2010). Receiving recommendations and providing feedback: The user-experience of a recommender system. In Proceedings 11th International Conference on E-commerce and web technologies (ECWeb), pp. 207-216. Springer.

Kokolakis, S. (2017). Privacy attitudes and privacy behaviour: a review of current research on the privacy paradox phenomenon. Computers \& Security, 64, 122-134.

Konstan, J.A., \& Riedl, J. (2012). Recommender systems: From algorithms to user experience. User Modeling and User-Adapted Interaction, 22(1), 101-123.

Li, H., Sarathy, R., Xu, H. (2011). The role of affect and cognition on online consumers' decision to disclose personal information to unfamiliar online vendors. Decision Support Systems, 51(3), 434-445.

Malhotra, N., Kim, S., Agarwal, J. (2004). Internet users' information privacy concerns (iuipc): The construct, the scale, and a causal model. Information Systems Research, 15(4), 336-355.

McCrae, R.R., \& Costa, P.T. (1987). Validation of the five-factor model of personality across instruments and observers. Journal of Personality and Social Psychology, 52(1), 81.

McCrae, R.R., \& John, O.P. (1992). An introduction to the five-factor model and its applications. Journal of Personality, 60(2), 175-215.

McNee, S.M., Riedl, J., Konstan, J.A. (2006). Being accurate is not enough: How accuracy metrics have hurt recommender systems. In CHI'O6 Extended abstracts on human factors in computing systems, pp. 1097-1101. ACM.

Mischel, W., Shoda, Y., Ayduk, O. (2007). Introduction to personality: Toward an integrative science of the person. John Wiley \& Sons.

Mook, D.G. (1983). In defense of external invalidity. American Psychologist, 38(4), 379.

Norberg, P., Horne, D., Horne, D. (2007). The privacy paradox: Personal information disclosure intentions versus behaviors. Journal of Consumer Affairs, 41(1), 100-126.

Perkins, A.M. (2014). The Positive Side of Negative Emotions, Guilford Publications. Anxiety as an Adaptive Emotion, pp. 37-54. Guilford Publications.

Rammstedt, B., Goldberg, L.R., Borg, I. (2010). The measurement equivalence of big-five factor markers for persons with different levels of education. Journal of Research in Personality, 44(1), 53-61.

Ray, S., Kim, S., Morris, J. (2014). The central role of engagement in online communities. Information Systems Research, 25(3), 528-546.

Rentfrow, P.J., Gosling, S.D., Jokela, M., Stillwell, D.J., Kosinski, M., Potter, J. (2013). Divided we stand: Three psychological regions of the United States and their political, economic, social, and health correlates. Journal of Personality and Social Psychology, 105(6), 996.

Ricci, F., Rokach, L., Shapira, B. (eds.) (2015). Recommender Systems Handbook, 2nd edn Springer.

Rich, B., LePine, J., Crawford, E. (2010). Job engagement: Antecedents and effects on job performance. Academy of Management Journal, 53(3), 617-635.

Ross, C., Orr, E.S., Sisic, M., Arseneault, J.M., Simmering, M.G., Orr, R.R. (2009). Personality and motivations associated with facebook use. Computers in Human Behavior, 25(2), 578-586.

Rossetti, M., Stella, F., Zanker, M. (2016). Contrasting offline and online results when evaluating recommendation algorithms. In Proceedings of the 10th ACM Conference on Recommender Systems, RecSys '16 (pp. 31-34). New York: ACM.

Servaas, M., Aleman, A., Marsman, J.B., Renken, R., Riese, H., Ormel, J. (2015). Lower dorsal striatum activation in association with neuroticism during the acceptance of unfair offers. Cognitive, Affective \& Behavioral Neuroscience, 15(3), 537-552. 
Smith, H., Dinev, T., Xu, H. (2011). Information privacy research: an interdisciplinary review. MIS Quarterly, 35(4), 989-1015.

Smith, H., Milberg, S., Burke, S. (1996). Information privacy: Measuring individuals' concerns about organizational practices. MIS Quarterly, 20, 167-196.

Son, J.Y., \& Kim, S.S. (2008). Internet users' information privacy-protective responses: a taxonomy and a nomological model. MIS Quarterly, 32(3), 503-529.

Stewart, K.A., \& Segars, A.H. (2002). An empirical examination of the concern for information privacy instrument. Information Systems Research, 13(1), 36-49.

Teng, C.I. (2008). Personality differences between online game players and nonplayers in a student sample. CyberPsychology \& Behavior, 11(2), 232-234.

Tkalcic, M., \& Chen, L. (2015). Personality and recommender systems. In Recommender systems handbook, pp. 715-739. Springer.

Tyler, T. (1994). Psychological models of the justice motive: Antecedents of distributive and procedural justice. Journal of Personality and Social Psychology, 67(5), 850-863.

Werthner, H., Klein, S., et al. (1999). Information technology and tourism: a challenging relationship. Springer-Verlag Wien.

Werthner, H., \& Ricci, F. (2004). E-commerce and tourism. Communications of the ACM, 47(12), 101-105.

Westin, A.F. (2003). Social and political dimensions of privacy. Journal of Social Issues, 59(2), 431-453.

Wisniewski, P., Xu, H., Lipford, H., Bello-Ogunu, E. (2015). Facebook apps and tagging: the trade-off between personal privacy and engaging with friends. Journal of the Association for Information Science and Technology, 66(9), 1883-1896.

Xu, H., Luo, X.R., Carroll, J.M., Rosson, M.B. (2011). The personalization privacy paradox: an exploratory study of decision making process for location-aware marketing. Decision support systems, 51(1), 42-52.

Xu, H., Teo, H.H., Tan, B.C., Agarwal, R. (2012). Research note-effects of individual self-protection, industry self-regulation, and government regulation on privacy concerns: a study of location-based services. Information Systems Research, 23(4), 1342-1363.

Zhao, H., \& Seibert, S.E. (2006). The big five personality dimensions and entrepreneurial status: a metaanalytical review. Journal of Applied Psychology, 91(2), 259-271. 